\title{
EL USO DEL LABORATORIO DE LA ENSEÑANZA DE LA MATEMÁTICA EN LA FORMACIÓN DE PROFESORES: Pasantía supervisada y colaboración.
}

\author{
Nayara Katherine Duarte Pinto \\ nayarakatherine@,hotmail.com \\ https://orcid.org/0000-0003-0008-0453 \\ Universidade Federal de Minas Gerais (UFMG) \\ Belo Horizonte, MG, Brasil \\ Keli Cristina Conti \\ keli.conti@gmail.com \\ https://orcid.org/0000-0001-5662-2923 \\ Universidade Federal de Minas Gerais (UFMG) \\ Belo Horizonte, MG, Brasil
}

Recibido: 31/10/2020 Aceptado: 02/02/2021

\begin{abstract}
Resumen
Este artículo comprende algunos de los aspectos más destacados de una tesis de maestría (Pinto, 2020). Pretendemos traer elementos de participación de uno de los estudiantes de licenciatura, que participó en el trabajo de campo de la investigación y de su profesora supervisora, con el enfoque en los usos del Laboratorio de Enseñanza de Matemáticas (LEM) de la Facultad de Educación (FaE) de la Universidad Federal de Minas Gerais (UFMG) en la formación de profesores en el contexto de prácticas supervisadas y colaboración entre los participantes. La investigación descrita se desarrolló con un enfoque cualitativo. Los encuentros de trabajo de campo para la planificación y evaluación del plan de clases se llevaron a cabo en el LEM FaE y el desarrollo del plan en una escuela pública a nivel federal. De esta forma, presentamos a lo largo del texto, detalles del contexto de la investigación. E1 marco teórico transcurre por algunas concepciones del Laboratorio de Enseñanza de las Matemáticas, pasantías supervisadas y colaboración. Concluimos con una comprensión que el LEM FaE puede ser un espacio que permita el intercambio de conocimientos, nuevos aprendizajes, experimentación, planificación, desarrollo y evaluación de actividades, notándose alcances en el ámbito de las pasantías supervisadas y colaboración entre los participantes.
\end{abstract}

Palabras claves: Laboratorio de Enseñanza de las Matemáticas. Pasantía Supervisada ${ }^{1}$. Colaboración. Formación de profesores de matemáticas. Educación Matemática.

\section{USO DO LABORATÓRIO DE ENSINO DE MATEMÁTICA NA FORMAÇÃO DE PROFESSORES: Estágio supervisionado e colaboração}

\begin{abstract}
Resumo
Este artigo compreende alguns destaques de uma dissertação de mestrado (Pinto, 2020). Neste artigo, tivemos o objetivo de trazer elementos da participação de um dos licenciandos, que
\end{abstract}

\footnotetext{
${ }^{1}$ En Brasil el término más utilizado es "Estágio Supervisionado", elegimos "Pasantía Supervisada" en español, pero también es posible encontrar similitudes como "Prácticas Integrales" y "Prácticas pedagogicas".
} 
participou do trabalho de campo da pesquisa, e de sua professora supervisora, com o foco nos usos do Laboratório de Ensino de Matemática (LEM) da Faculdade de Educação (FaE) da Universidade Federal de Minas Gerais (UFMG) na formação de professores no âmbito do estágio supervisionado e colaboração entre os participantes. A pesquisa descrita foi desenvolvida numa abordagem qualitativa. Os encontros do trabalho de campo, para planejamento e avaliação do plano de aula ocorreram no LEM FaE, e o desenvolvimento, do plano, em uma escola pública de âmbito federal. Dessa maneira, apresentamos ao longo do texto detalhes do contexto da pesquisa. O referencial teórico perpassa por algumas concepções do Laboratório de Ensino de Matemática, estágio supervisionado e colaboração. Finalizamos com uma compreensão de que o LEM FaE pode ser um espaço que possibilita trocas de conhecimento, novas aprendizagens, experimentações, planejamentos, desenvolvimentos e avaliações de atividades, sendo notado alcances no âmbito do estágio supervisionando e colaboração entres os participantes.

Palavras-chave: Laboratório de Ensino de Matemática. Estágio supervisionado. Colaboração. Formação de Professores de Matemática. Educação Matemática.

\title{
THE USE OF MATHEMATICS TEACHING LABORATORY IN TEACHER TRAINING: Supervised internship and collaboration
}

\begin{abstract}
This article comprises some highlights of a master's dissertation (Pinto, 2020). In this article, we have the objective of bring elements of the participation of one the undergraduate, who participated in the research field work, and of your surpervising teacher the focus of the uses of the Laboratory of Teaching of Mathematics (LEM) of the Faculty of Education (FaE) of the Federal University of Minas Gerais (UFMG) in the training of teachers in scope the supervised internship and collaboration between the participants. The research described was developed in a qualitative approach. The meetings for planning and evaluating the lesson plan in LEM FaE of the institution and the development, of the plan, in a federal public school. In this way, we present throughout the text details of the research context. The theoretical framework, runs through some conceptions of the Mathematics Teaching Laboratory, supervised internship and collaboration. We end with an understanding that LEM FaE of the institution can be a space that enables knowledge exchange, new learning, experimentation, planning, development and evaluation of activities, being noticed reaches in the scope within the scope of supervised internship and collaboration between the participants.
\end{abstract}

Keywords: Mathematics Teaching Laboratory. Supervised Internship. Collaboration. Mathematics Teacher TraininGustavo Mathematics education.

\section{Introducción}

En este estudio divulgamos algunos aspectos destacados de la disertación (Pinto, 2020) de un Programa de Maestría Profesional en la línea de la Educación Matemática.

Luego de las reflexiones e inquietudes de la investigadora al cursar el Laboratorio de Enseñanza de las Matemáticas de la Facultad de Educación (LEM FaE) y, al leer el trabajo de 
Lorenzato (2009), surgió el interés por realizar la investigación, que tenía como objetivo general analizar los alcances y limitaciones del uso de LEM FaE como apoyo en la planificación de clases a ser desarrolladas dentro de la pasantía supervisada de los estudiantes de licenciatura en Matemáticas.

Nuestro objetivo fue traer elementos de la contribución de uno de los estudiantes de licenciatura, que participó en el trabajo de campo de la investigación, y de su profesora supervisora, con un enfoque en los usos de LEM FaE en la formación de profesores en el ámbito de la pasantía supervisada y de la colaboración.

De esta forma, organizamos este trabajo en cuatro secciones. En la primera "contexto de la investigación" presentamos elementos para que el lector tenga una mejor comprensión de la metodología de investigación y el plan de trabajo. En la segunda sección "marco teórico", repasaremos los temas principales de este artículo, que son algunas concepciones del LEM, la pasantía supervisada y la colaboración. En la tercera sección "Participación del trabajo de campo del Licenciatario Gustavo y la Profesora Mariana" se muestra una breve descripción del estudiante de Licenciatura y de su supervisora. Posteriormente, tendremos los apartados que describen los encuentros en el LEM FaE, las Evaluaciones del estudiante de licenciatura Gustavo y la Profesora Mariana sobre su participación en el trabajo de campo, y una discusión de los alcances y las limitaciones del uso del LEM FaE, en lo que se refiere a la pasantía supervisada y la colaboración. Finalmente, la última sesión estará destinada para discutir sobre algunas consideraciones.

\section{El contexto de la investigación}

La investigación se desarrolló a través de un enfoque cualitativo, en la concepción de Bogdan y Biklen (1994). Esto se debe a que creemos que, en enfoques de esta naturaleza, es posible describir y analizar mejor la investigación y sus acciones, como lo proponen estas las cinco características:

1) En la investigación cualitativa la fuente directa de datos es el ambiente natural, siendo el investigador el principal instrumento [...];

2) La investigación cualitativa es descriptiva [...];

3) Los investigadores cualitativos están más interesados en el proceso en sí que en los resultados o productos [...];

4) Los investigadores cualitativos tienden a analizar sus datos de forma inductiva [...];

5) El significado es de vital importancia en el enfoque cualitativo (Bogdan; Biklen, 1994, p. 47-50). 
Partiendo de los principios del enfoque cualitativo, tuvimos como pregunta guía de la investigación: ¿Cuáles son los alcances y limitaciones del LEM FaE, como soporte en la planificación de clases a ser desarrolladas en el ámbito de la pasantía supervisada de los estudiantes de licenciatura en matemáticas? Así, la invitación para participar en la investigación fue a los estudiantes de la licenciatura en formación, que estaban cursando la disciplina de la Pasantía Supervisada II, en el segundo semestre del 2018, para preparar de manera colaborativa los planes de clases en el LEM FaE a ser desarrollados en el ámbito de la pasantía por parte de los estudiantes de la licenciatura.

Para lograr este objetivo, y con base a Bogdan y Biklen (1994), los instrumentos de registro de la investigación fueron: Cuestionario de Identificación de los Participantes; Registro de audio de las actividades desarrolladas en los encuentros; Diario de campo, elaborado por la investigadora; Entrevista semiestructurada con los participantes de la investigación; y, Registro de intercambios de correos electrónicos y mensajes por el aplicativo de Whats App con los participantes de la investigación.

Poco después de la aprobación del Comité de Investigación en Humanos del Ministerio de Salud, por parte del Sistema Nacional de Información sobre Ética en Investigación que incluye a los Seres Humanos (SISNEP), formalizamos la alianza con la profesora responsable, Samira Zaidan, para las disciplinas de Pasantías Supervisadas en 2018, época del trabajo de campo, para que ella interviniera en nuestra invitación a los estudiantes de licenciatura para que ellos participaran de esta investigación.

Durante una reunión previa con la profesora responsable por las asignaturas de la pasantía, de común acuerdo, resolvimos que la investigadora participaría en las clases de la disciplina para que los estudiantes de licenciatura ganaran confianza y se identificaran con la investigadora. Ya con la intención de comenzar a desarrollar los principios de colaboración contenidos en Fiorentini (2013), que son: voluntariedad, identidad y espontaneidad; liderazgo compartido y corresponsabilidad; apoyo, respeto mutuo y la reciprocidad del aprendizaje. Queremos resaltar que, en vista del objetivo de la investigación y las limitaciones del tiempo, lo que sucedida durante las clases de la Pasantía Supervisada II no fue considerado en la investigación, ni se investigó más a fondo las instituciones receptoras de los licenciados y sus estudiantes. 
El plan de trabajo de la investigación comprendió tres fases que incluyeron la planificación (investigador, estudiantes de licenciatura y profesor supervisor-opcional ${ }^{2}$ ), el desarrollo (por los estudiantes de licenciatura) y la evaluación de los planes de clases (investigadora, estudiantes de licenciatura y profesor supervisor-opcional) después de la realización de las clases junto a los estudiantes de la escuela. Concebimos que las fases funcionarían como un ciclo, que podría reiniciarse según las demandas de los participantes de la investigación en la planificación nuevas clases.

Las reuniones para el trabajo de campo serían en momentos extra-clase en el LEM FaE. Luego del inicio del contacto con los estudiantes de licenciatura, tuvimos la adhesión de cinco de ellos, o sea, participaron del trabajo de campo: Carolina, Eric, Fabiana, Gustavo y Leandro, y la profesora Mariana, quien supervisaba al estudiante Gustavo. Algunos de los otros estudiantes manifestaron interés, pero no tuvieron tiempo para los encuentros.

De forma general ${ }^{3}$, los estudiantes licenciatura eran jóvenes con poca o ninguna experiencia en la docencia de las matemáticas. En cuanto al nivel educativo en que ellos realizaban la pasantía, tenían, la Educación para Jóvenes y Adultos (Años iniciales), de la Secundaría básica (Últimos Años (octavo y noveno)) y de la Educación Media (décimo, onceno y doce grado).

Los encuentros fueron periódicos, de acuerdo con la disponibilidad de los estudiantes de licenciatura, de la investigadora y del LEM FaE; para la investigación, discusión, planificación, estudio y pruebas, tuvieron como objetivo elaborar los planes de clase de los estudiantes. Después de las primeras reuniones, y teniendo en cuenta las particularidades de cada estudiante, con un previo plan de clase elaborado, hicimos una invitación a los profesores supervisores de la Pasantía Supervisada de los estudiantes de licenciatura, para que ellos, si quisiesen, pudieran contribuir al proceso de elaboración final y evaluación de los planes de clases en el LEM FaE.

Teniendo en cuenta el objetivo de este artículo, pasamos a continuación a comentar algunas concepciones del Laboratorio de Enseñanza de las Matemáticas, de la Pasantía Supervisada y de la colaboración.

\footnotetext{
${ }^{2}$ La participación de los profesores supervisores de la asignatura de la pasantía supervisada II fue gratuita y un profesor aceptó participar en la investigación.

${ }^{3}$ Los mejores detalles de cada participante y de los acontecimientos del trabajo de campo, incluida la descripción de los encuentros, evaluación, análisis y planes de clases están disponibles en el capítulo 5 de la investigación de Pinto (2020).
} 


\section{Referencial Teórico}

Para este estudio elegimos el término de Laboratorio de Enseñanza de las Matemáticas (LEM), considerando que existen varias posibilidades de nomenclaturas, tales como: Laboratorio de Enseñanza de las Matemáticas, Laboratorio de estudios, Laboratorio de Matemática, Laboratorio de Educación Matemática, Sala de Matemática, entre otros. Además, pensamos que las formas de uso de estos espacios tienen un protagonismo mayor que sus denominaciones. Ahora, pasamos por los temas principales de este estudio, que son algunas concepciones del LEM, la Pasantía Supervisada y la colaboración.

\section{Algunas concepciones del LEM}

Como base inicial, mostraremos las diferentes concepciones del uso del LEM presentes en Lorenzato (2009) y, siempre que sea posible, las reforzaremos con las experiencias, estudios de otros autores y complementándolas con otras visiones del LEM, con el fin de mostrar algunas evoluciones de estos usos.

La primera de ellas sería la idea de que el LEM "podría ser un lugar para almacenar materiales esenciales, haciéndolos accesibles para las clases" (LORENZATO, 2009, p. 6). En este caso, podemos citar, como materiales esenciales, publicaciones en el área (libros, libros didácticos, revistas, manuales), materiales visuales y auditivos como: CD y DVD, juegos, instrumentos de medida, calculadoras, modelos estáticos y dinámicos, material didáctico industrializados, materia prima para la elaboración de materiales didácticos como: tijeras, papeles, pegamentos, hilo, botones, alambres, entre otros.

Esta concepción de laboratorio, o una característica de este, no implica necesariamente la existencia de una sala de clase separada. Podría ser, por ejemplo, un armario o una caja. De esta forma, Rodrigues (2012) destaca que:

Queda implícito la idea de que las clases de Matemáticas no se desarrollarán en el espacio físico de este laboratorio, estando el mismo restricto al apoyo y soporte, a través de sus materiales manipulables, para las actividades experimentales que se desarrollarán por el docente fuera de este entorno (p. 60).

Además, la autora destaca que el uso del LEM de esta manera es similar al de una biblioteca, lo que posibilita el acceso de estudiantes y profesores a estos materiales para ayudar en la construcción del conocimiento. Por tanto, Rodrigues (2012) considera que "esto sucede a medida que esto pone a disposición de sus usuarios una colección de libros, 
materiales didácticos diversificados que contribuirán al desarrollo de las actividades prácticas" (p. 60).

Desde otra perspectiva, Lorenzato (2009) muestra que, al volver a discutir esta primera concepción del LEM, este se vuelve:

Un lugar de la escuela reservado preferiblemente no solo para las clases regulares de matemáticas, sino también para responder a las preguntas de los estudiantes; para que los profesores de matemáticas planifiquen sus actividades, ya sean clases, exposiciones, Olimpiadas, entre otras, para discutir sus proyectos, tendencias e innovaciones; un lugar para la creación y desarrollo de actividades experimentales, incluyendo la producción de materiales de instrucciones que puedan facilitar la mejora de la práctica pedagógica (Lorenzato, 2009, p.6).

Conseguimos percibir, en esta mirada sobre el LEM, que este tiene un papel muy importante, en cuanto al espacio, tanto para las clases de matemáticas como para la planificación de actividades. En línea con esta idea, el estudio de Justo (2015) destaca que:

Probablemente, la existencia de este espacio organizado en la escuela, puede contribuir a un aprendizaje significativo, ya que es imposible definir actividades de enseñanza estandarizadas que satisfagan las necesidades de todos los estudiantes, por tanto, tornándose necesario, diversificar las estrategias y el uso de materiales, además del libro didáctico (Justo, 2015, p. 22-23).

La tercera concepción, que consideramos sea un nivel más amplio del LEM, presente en Lorenzato (2009), es la posibilidad de que el LEM sea:

(...) una sala-ambiente para estructurar, organizar, planificar y hacer realidad el pensamiento matemático, es un espacio para facilitar, tanto al alumno como al profesor, cuestionar, conjeturar, buscar, experimentar, analizar y concluir, en definitiva, aprender y principalmente aprender a aprender (Lorenzato, 2009, p. 7).

Si reflexionamos sobre esta definición, es como si existieran nuevos alcances para el LEM, que van más allá de sus materiales o espacio físico, relacionados con la estructuración del conocimiento de los involucrados. Así, Oliveira (2017, p. 45) expresa que "en este enfoque, el LEM pretende, en el ámbito escolar, ayudar al proceso de enseñanza-aprendizaje, en lo que respecta a desmitificar y facilitar la enseñanza de las Matemáticas”.

Durante los estudios de investigación, conocemos algunas experiencias de los usos y posibilidades del LEM, algunas en la Educación Básica, otras en Educación Superior. Con el fin de relacionarlas con las experiencias y estudios de esta investigación, coincidimos con el concepto presentado por la autora Oliveira (2017), de que “el Laboratorio de Enseñanza de las Matemáticas es un espacio de hacer y que favorece la reflexión continua del estudiante y el profesor, de lo qué se hace, para qué y cómo se hace, con el fin de proporcionar una enseñanza 
comprensiva" (p. 37). Este concepto, de espacio para hacer y reflexionar, puede estar presente en las asignaturas de Pasantías Supervisadas, que analizaremos a continuación.

\section{Pasantía supervisada}

El uso del espacio del LEM FaE está muy unido a las clases de Pasantías Supervisadas, en nuestro caso la asignatura de Pasantías Supervisadas II, y, en relación con los objetivos de la investigación, comenzamos a conversar un poco sobre algunas concepciones, presentes en el trabajo de Pimenta y Lima (2012), relacionado con estas clases, común en los cursos de licenciatura.

Inicialmente, las autoras destacan que, a menudo, las asignaturas de pasantías son vistas como la parte práctica de los cursos de licenciatura, siendo opuestas a las asignaturas teóricas. Sin embargo, en los argumentos de las autoras, por parte de los planos curriculares, las asignaturas, muchas veces son aisladas entre sí, y optan por cambiar la denominación de teoría para "saberes disciplinar en cursos de formación" (Pimenta; Lima, 2012, p. 33). Además, es preocupante cuando Pimenta y Lima (2012) discuten el hecho de que las asignaturas de estos currículos asumen "casi una total autonomía en relación con el campo de actuación de los profesionales y, especialmente, al significado social, cultural, humano del desempeño profesional.” (p. 33-34).

Estos argumentos preliminares sirven de introducción a la presentación de los cuatro conceptos de pasantía (Pimenta; Lima, 2012) siendo estas: práctica como imitación de modelos; práctica como herramienta técnica; pasantía como aproximación a la realidad y la actividad teórica; prácticas como investigación. Traemos, a partir de este momento, una síntesis de cada una de estas concepciones, con el fin de comprenderlas mejor.

La primera concepción, la práctica como imitación de modelos, surge de la idea de que se aprende imitando lo que fue observado, es decir, bastaría con reproducir lo aprendido previamente al enseñar. Para Pimenta y Lima (2012):

La suposición de esta concepción es que la realidad de la enseñanza es inmutable y los estudiantes que asisten a la escuela también lo son. Idealmente concebidos, correspondería a la escuela enseñarlos, según la tradición. Por tanto, no es necesario considerar las transformaciones históricas y sociales resultantes de los procesos de democratización del acceso, la cual trajo para la escuela nuevas demandas y realidades sociales, con la inclusión de alumnos hasta ahora marginados del proceso escolar y los procesos de transformación de la sociedad, de sus valores y las características que adquieren los niños y jóvenes (p. 35-36). 
Según esta perspectiva, Pimenta y Lima (2012), la pasantía se resumiría en la observación e imitación de los modelos que los profesores utilizan en las clases. De esta manera, quedaría reducido a observar la sala de clases sin hacer ninguna observación o reflexión sobre la realidad social de los involucrados.

A su vez, la segunda concepción, la práctica como instrumentación técnica, está relacionada, según Pimenta y Lima (2012), con el aprendizaje de las técnicas de la profesión de profesor, como ocurre en cualquier otra carrera como odontólogo y médico, por ejemplo. Si hiciéramos un análisis superficial, tendríamos una mirada positiva sobre esta concepción, pero esto esconde y refuerza, como destacan las autoras, algunos discursos y acciones culturales que son negativas, tales como "la práctica por la práctica" y "quién sabe hace, quien no sabe enseña”. Esto ocurre, de acuerdo con las autoras, porque existe una comprensión de pasantía, como "actitudes dicotómicas en que la teoría y la práctica son tratadas de forma aislada, lo que genera errores graves en los procesos de formación profesional” (Pimenta; Lima, 2012, p. 37).

Dicho esto, las actividades de pasantías, en este concepto, se enfocan en los momentos de práctica con el fin de aprender el "cómo hacer" y desarrollar las habilidades necesarias para el desempeño de la profesión. Además, Pimenta y Lima (2012) señalan que los cursos que adoptan esta concepción provocando "distanciamiento de la vida y el trabajo concreto que se da en las escuelas, una vez que las asignaturas que integran los cursos de formación no establecen los vínculos entre los contenidos que se desarrollan y la realidad en la que se desarrolla la enseñanza" (p. 39).

La tercera concepción, la pasantía como aproximación de la realidad y la actividad teórica, parte de una visión, que las autoras presentan en detalle minucioso, de que "la pasantía es teoría y práctica (y no teoría $o$ práctica)” (Pimenta; Lima, 2012, p. 41, cursiva agregada), así, una forma de encerrar con la comprensión de la existencia dicotómica entre actividad teórica y práctica, considerando que, comprender bien la relación entre la teoría y práctica posibilita nuevas y mejores experiencias en las asignaturas de la pasantía.

En resumen, en esta tercera concepción, las actividades de pasantías estarían comprometidas con el contexto escolar, enfocándose en el entorno social en el que ellas ocurren, para que la práctica sea efectivamente relevante para los involucrados y que las teorías auxilien en la construcción y reflexión de las intervenciones. Además, las autoras debaten un poco sobre el papel de los profesores supervisores que analizan esta concepción. 
Es necesario que los profesores supervisores de las pasantías, lleven a cabo en el colectivo, con sus compañeros y alumnos, esta aproximación a la realidad, para analizarla y cuestionarla críticamente, a la luz de las teorías. Este recorrido conceptual, ciertamente será un camino para proponer nuevas experiencias (Pimenta; Lima, 2012, p. 45).

Finalmente, en la cuarta concepción, las autoras discuten sobre la pasantía como investigación, siendo esta una alternativa para la formación de profesores. Esta concepción consta de dos características:

Por un lado, la movilización de investigaciones que permitan la ampliación y análisis de los contextos donde se realizan las pasantías; por otro lado, y en particular, se traduce en la posibilidad de que los practicantes desarrollen la postura y habilidades del investigador a partir de situaciones de la pasantía, desarrollando proyectos que les permitan al mismo tiempo comprender y problematizar las situaciones que observan (Pimenta; Lima, 2012, p. 46).

Por tanto, la cuarta concepción sería la forma más amplia entre las presentadas en Pimenta y Lima (2012), ya que se indican aportes más esclarecedores ${ }^{4}$ para el cambio en la formación de profesores. Ante esto, se enfatiza, respecto a los cambios que se desean obtener, "que se busquen nuevos conocimientos en la relación entre las explicaciones existentes y los datos nuevos que la realidad impone y que son percibidos en la actitud investigativa" (Pimenta; Lima, 2012, p. 46).

Dado lo anterior, consideramos que las asignaturas de la pasantía se pueden articular con el LEM, pero deseamos, desde su elaboración, que la investigación permitiera que el espacio del LEM FaE fuera un soporte tanto para los momentos de estudios teóricos como para las actividades prácticas, con el fin de aproximarse a la tercera y cuarta concepción propuesta por Pimenta y Lima (2012), abarcadas en las asignaturas de Pasantías Supervisada del curso de Licenciatura en Matemáticas de la UFMG y que son las concepciones que consideramos más adecuadas en la formación del profesor.

Continuaremos en la próxima sesión abordando otra intención del estudio, que fue propiciada en un entorno colaborativo del LEM FaE.

\footnotetext{
${ }^{4}$ Según las autoras, cuando los cursos siguen una concepción que disocia la teoría de la práctica, las experiencias de las asignaturas de pasantía se empobrecen. Por otro lado, cuando adoptan una concepción en la que la teoría y la práctica están juntas, ganan más alcance y claridad, por eso utilizan el término más esclarecedor.
} 


\section{Colaboración}

La colaboración fue un tema que impregnó este estudio, por aspirar tener como contexto durante el trabajo de campo, un ambiente de colaboración entre las investigadoras y los participantes. Por tanto, en este tópico, con la ayuda de Fiorentini (2013), traeremos algunos argumentos sobre las singularidades de realizar una investigación en un entorno colaborativo 5 .

Según Fiorentini (2013), los “aspectos característicos y constitutivos del trabajo colaborativo" (p. 58), que él presencia en sus estudios y experiencias con grupos colaborativos, son: voluntariedad, identidad y espontaneidad; liderazgo compartido y corresponsabilidad; apoyo, respeto mutuo y la reciprocidad del aprendizaje.

El primero se caracteriza por la forma voluntaria de cómo se da la búsqueda de las personas para participar de un grupo colaborativo, y surge a partir de su propia voluntad e identificación con los ideales del grupo. Como resultado, Fiorentini (2013) enumera algunas de las razones por las que los profesores buscan a los grupos de colaboración:

Buscar apoyo y colegas para comprender y enfrentar los problemas complejos de la práctica profesional; enfrentar conjuntamente los desafíos de la innovación curricular en la escuela; desarrollar proyectos de innovación tecnológica, como la incorporación de tecnologías de la información y la comunicación (computadores, internet, videos, etc.) en la práctica escolar; buscar su propio desarrollo profesional; Desarrollar investigaciones sobre la propia práctica, entre otros (Fiorentini, 2013, p. 60).

Respecto al liderazgo compartido y la corresponsabilidad, Fiorentini (2013) enfatiza que, para que un grupo alcance una etapa de armonía en la toma de decisiones y división de tareas, puede llevar un tiempo, pero cuando los participantes del grupo lo comprenden bien, estos momentos sucederán con cada uno, eligiendo sus tareas de forma voluntaria y espontánea. Otro punto importante destacar es el hecho de que puede haber algunos conflictos y variaciones de liderazgo en determinados momentos, lo cual es algo natural. En resumen, todas las actividades que serán realizadas por el grupo serán responsabilidad de todos y se distribuirán a voluntad de cada uno.

Así, algunos factores que contribuyen a la existencia del respeto y apoyo mutuo en las relaciones que se establecen en estos grupos, según el trabajo de Fiorentini (2013), son:

El grupo, en estos casos, ha expresado, por un lado, un profundo respeto por los conocimientos y experiencias conceptuales que cada profesor aporta a los

\footnotetext{
${ }^{5}$ El autor también discute sobre lo qué es investigar en colaboración, pero ese no fue nuestro caso. Para obtener
} más información, consulte Fiorentini (2013). 
encuentros, así como en relación a sus dificultades y posibles fracasos, y, por otro, dar un apoyo emocional e intentando encontrar colaborativamente soluciones a los problemas (Fiorentini, 2013, p. 64).

En conclusión, deseamos crear un contexto que permitiera, entre estudiantes de licenciatura y la investigadora (y profesores, en caso que fuese posible), un entorno que sobresalga en los elementos señalados por Fiorentini (2013): voluntariedad, identidad y espontaneidad; liderazgo compartido y corresponsabilidad; apoyo, respeto mutuo y reciprocidad del aprendizaje. Creyendo que esto contribuiría con la formación de los estudiantes de licenciatura y con la investigación, y estaría en sintonía con el LEM y sus usos.

En la próxima sesión, traeremos más detalles de la participación en el trabajo de campo de investigación de uno de los estudiantes de la licenciatura y de la profesora que supervisó la pasantía.

\section{La participación en el trabajo de campo del estudiante de licenciatura Gustavo y de la profesora Mariana}

El estudiante de licenciatura Gustavo es oriundo de Belo Horizonte y, en el momento de la investigación, tenía 23 años. De formación en escuela pública, se encontraba en su primer pregrado, habiéndose incorporado en el año 2014. Él trabajaba como monitor en exámenes de acceso a la universidad y como profesor de clases particulares. Por la información contenida en su cuestionario de identificación, él no consideraba esas actividades remuneradas como experiencia docente en Matemática.

En cuanto a la Pasantía Supervisada, se desempeñó en los grupos de clases de $1^{\circ}$ y $2^{\circ}$ año del bachillerato (décimo y onceno grado), con un promedio cada uno de 35 estudiantes, siendo una escuela técnica y pública a nivel federal. El estudiante Gustavo consideró la institución como una estructura de porte medio y tuvo como supervisora a la profesora Mariana Lima, quien también aceptó la invitación para participar del trabajo de campo.

Según las informaciones contenidas de su cuestionario, la profesora Mariana tenía 25 años en el momento de la investigación y es oriunda de Divinópolis, una ciudad del interior de Minas Gerais. Ella viene de una escuela pública, graduada en Licenciatura en Matemáticas por la UFMG y estaba haciendo un posgrado en el área de la Educación Matemática. Fue su primera experiencia profesional como docente de Matemáticas y como profesora sustituta, desde mayo del 2017, en una escuela técnica y pública a nivel federal de Belo Horizonte, 
impartiendo clases para todos los años de la enseñanza media (décimo, onceno y doce grados). Consideró esta institución como una estructura de gran porte. Además, recibió a practicantes con cierta frecuencia e informó que el estudiante de Licenciatura Gustavo era el octavo pasante que ella supervisaba.

La profesora Mariana consideró que las asignaturas de las Pasantías Supervisadas fueron fundamentales para su permanencia en la Universidad porque era un espacio que le permitió discutir la enseñanza de las Matemáticas en la educación básica. Ella informó que su primer contacto con el LEM FaE, fue en el segundo semestre del año 2018, sucedió cuando ella estaba de pasantía docente, y la asignatura que ella acompañaba ocurría en el LEM FaE.

En el siguiente texto seguiremos con los siguientes apartados ${ }^{6}$ : descripción de las reuniones en el LEM FaE; las evaluaciones del estudiante de licenciatura Gustavo y la profesora Mariana sobre sus participaciones en el trabajo de campo; y alcance y limitaciones del uso del LEM FaE: pasantías supervisada y colaboración.

\section{Descripción de los encuentros en el LEM FaE}

En cuanto a las reuniones, para la planificación de las clases, como el estudiante de licenciatura Gustavo estaba trabajando en cinco lugares diferentes en el segundo semestre del año 2018, él disponía de media hora antes de los encuentros de la Pasantía Supervisada II, pero, como el propósito de la investigación era actuar de acuerdo con la disponibilidad de los involucrados, seguimos trabajando con esta limitación para los encuentros.

Nuestro primer encuentro fue el día 24 de septiembre del año 2018. Previo al encuentro, el estudiante de licenciatura Gustavo había comentado que el público objetivo de la clase a planificar estaba formado por estudiantes de segundo año de la enseñanza media (onceno grado), y la profesora supervisora Mariana recomendó que el tema de la clase fuera introducir el contenido de las funciones seno y coseno. Es decir, posibilitar la transición del ciclo trigonométrico para las funciones seno y coseno. El encuentro consistió en conocer mejor los materiales que el LEM FaE tenía sobre el tema sugerido por la profesora.

\footnotetext{
${ }^{6}$ Dada la limitación de páginas del artículo, algunas de las informaciones no se pudieron disponibilizar en ese momento, como la evolución del plan de clase y de la guía de actividad de los grupos, pero el capítulo 5 de la disertación Pinto (2020) contiene la descripción completa de la participación en el trabajo de campo de la investigación del estudiante de Licenciatura Gustavo y la Profesora Mariana, además de los demás participantes.
} 
Las siguientes figuras muestran los materiales disponibles en el momento de la investigación sobre el tema previsto en el LEM FaE. La figura 1 es la imagen del ciclo trigonométrico con triángulos en E.V.A.

Figura 1. Ciclo Trigonométrico con triángulos en E.V.A.

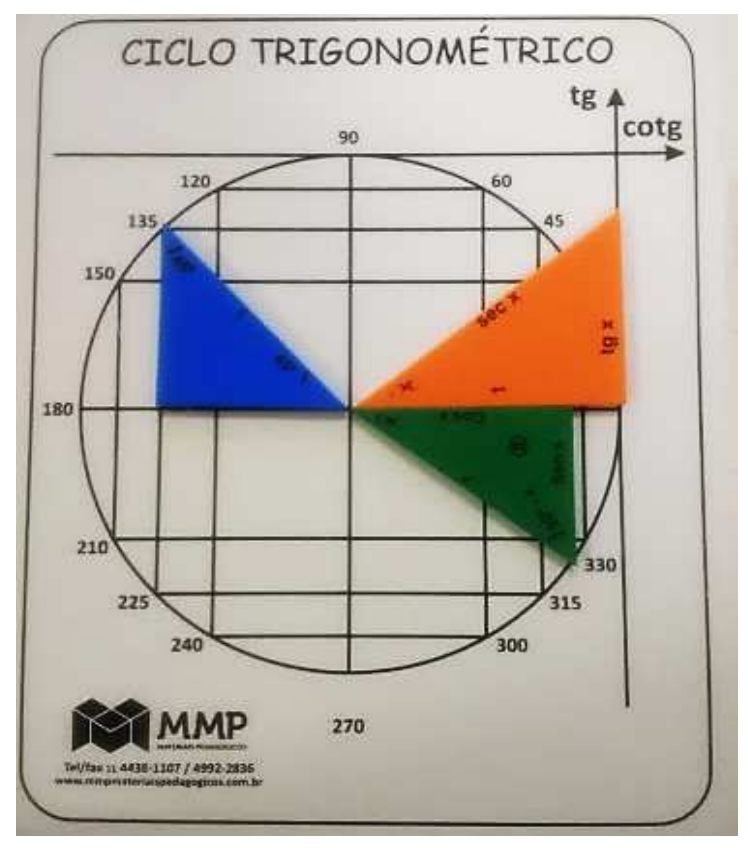

Fuente: Archivo personal de los investigadores (2020)

En la Figura 2, está el kit de ciclo trigonométrico en metal con triángulos magnetizados que sirve de ayuda al profesor. Tiene un tamaño que permite una mejor visualización por parte de los alumnos, y por estar magnetizado permite más practicidad y agilidad, que dibujar el mismo ciclo en el tablero. 
Figura 2. Ciclo trigonométrico en metal con triángulos magnetizados

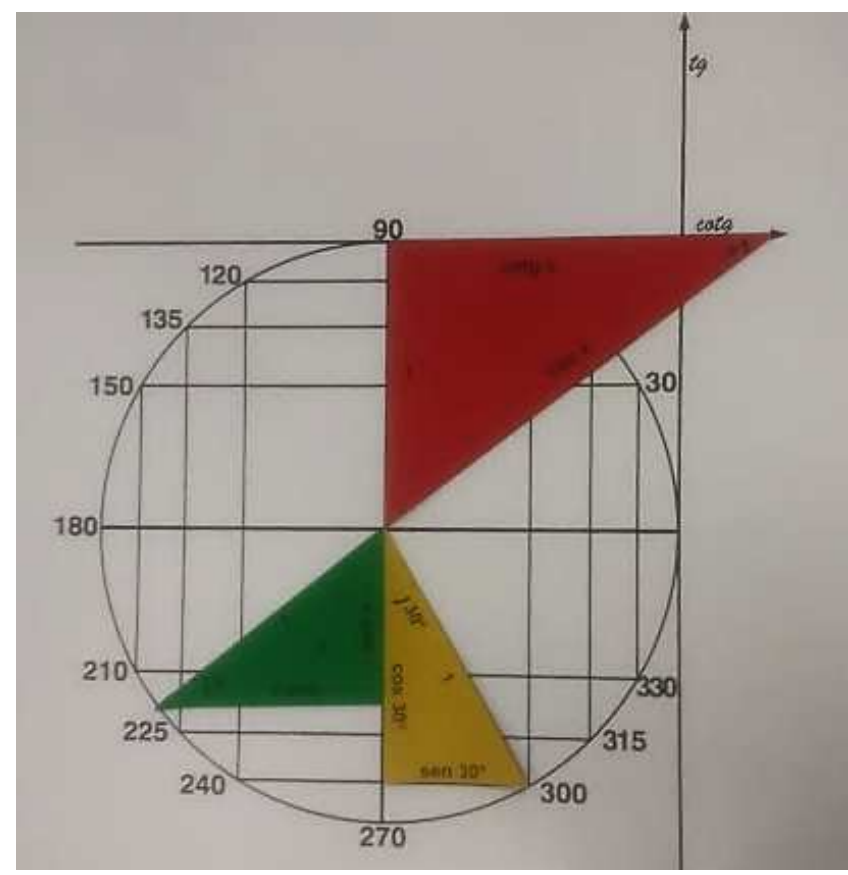

Fuente: Archivo personal de los investigadores (2020)

Finalmente, en la Figura 3, presentamos la imagen del Juego Trigonométrico Mándala, disponible en el LEM FaE.

Figura 3. Juego trigonométrico mándala.

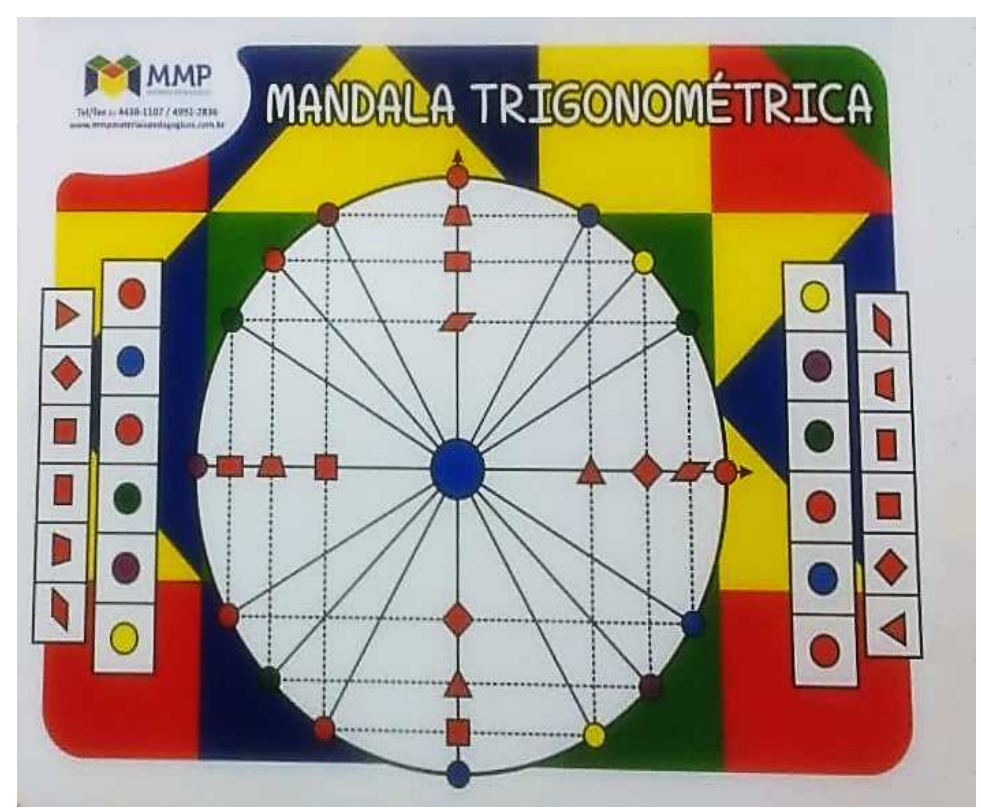

Fuente: Archivo personal de los investigadores (2020)

De esta forma, analizamos cada uno de los materiales para conocerlos mejor, y el estudiante de licenciatura Gustavo les tomó fotografías para mostrárselas a la profesora 
Mariana. Además, también asistimos a dos videos que el estudiante de licenciatura Gustavo encontró en una búsqueda de la construcción de las funciones seno y coseno en el software GeoGebra. Al final de la primera reunión, concluimos que los materiales del LEM FaE no serían útiles para el tema propuesto por la profesora Mariana. Aun así, el estudiante de licenciatura Gustavo conversaría con ella sobre otras posibilidades que ofrecían los materiales y sobre la opción de utilizar el GeoGebra. En este momento, el LEM FaE sirvió para analizar y reflexionar sobre los materiales en relación a la temática de la clase, haciendo referencia a lo propuesto por Pimenta y Lima (2012) en la tercera concepción de la pasantía al resaltar una relación entre teoría y práctica.

Debido a los compromisos laborales del estudiante de licenciatura Gustavo, el segundo encuentro se realizó apenas el 8 de octubre del año 2018, pero, en ese intervalo, se intercambiaron mensajes vía WhatsApp. En una de estas conversaciones, el estudiante de licenciatura comentó que la profesora Mariana había optado por cambiar el tema de la clase, y dejó como nuevo tema la introducción del contenido del Ciclo Trigonométrico, y así posibilitó el uso de materiales del LEM FaE.

Volviendo al segundo encuentro, estuvieron presentes el estudiante de licenciatura Gustavo, la profesora Mariana y la investigadora. El encuentro duró unos 15 minutos. La profesora pudo conocer mejor los materiales sobre el tema, ya que ella los había conocido solamente a través de imágenes. Durante la conversación, llegamos al consenso de que sería importante utilizar el Kit de Ciclo Trigonométrico en metal con triángulos magnetizados para introducir el tema y que, a partir del Kit de Ciclo Trigonométrico con triángulos en EVA, haríamos una guía que permitiera a los estudiantes manipular el material. Así, utilizando el Kit de Ciclo Trigonométrico con triángulos en EVA, se elaboró el material necesario para el uso de la guía por parte de todos los estudiantes de la clase. En este encuentro, el LEM FaE posibilitó un debate entre los involucrados para la planificación de las clases, y fue posible percibir elementos de colaboración, en las decisiones y divisiones de tareas, propuestas por Fiorentini (2013), como, por ejemplo, el respeto mutuo, liderazgo compartido y corresponsabilidad.

A pesar de la brevedad del encuentro, este fue positivo, principalmente por la presencia de la profesora Mariana $\mathrm{y}$, debido a la baja disponibilidad del estudiante de licenciatura Gustavo, la profesora acordó ser parte de las actividades del pasante y la confección de los 
materiales en el LEM FaE que se utilizarían en el desarrollo del plan de clases. Además, el estudiante de licenciatura Gustavo quedó responsable por la redacción de la guía de clase, como fue discutido en el encuentro.

El tercer encuentro de planificación, que tuvo lugar el 19 de octubre del año 2018, debía realizarse en otra sala de la institución, ya que el LEM estaba reservado para otra clase del día. En vista de esto, tomamos los materiales del LEM FaE que podrían usarse para el encuentro, entre ellos: tijeras, transportador, compás, algunos tipos de papel en diferentes colores, E.V.A, en diferentes colores, regla, Kit de ciclo trigonométrico con triángulos en EVA, Kit de ciclo trigonométrico de metal con triángulos magnetizados. En este encuentro, los recursos del LEM FaE sirvieron de apoyo para la producción de materiales que serían utilizados en la clase y, nuevamente, se percibieron elementos de colaboración, definidos por Fiorentini (2013), como, por ejemplo, la voluntariedad, respeto mutuo, liderazgo y corresponsabilidad entre los presentes.

Conversamos sobre las unidades de medida y el patrón de los triángulos. Además, escogimos como molde lo que estaba más perfecto. Las guías fueron impresas por la institución receptora, y el estudiante de licenciatura, en conjunto con la investigadora se encargaron de la preparación e impresión necesaria del material. Las figuras a continuación muestran las fotos del material que se confeccionó en ese encuentro.

Figura 4. Ciclo trigonométrico con triángulo A

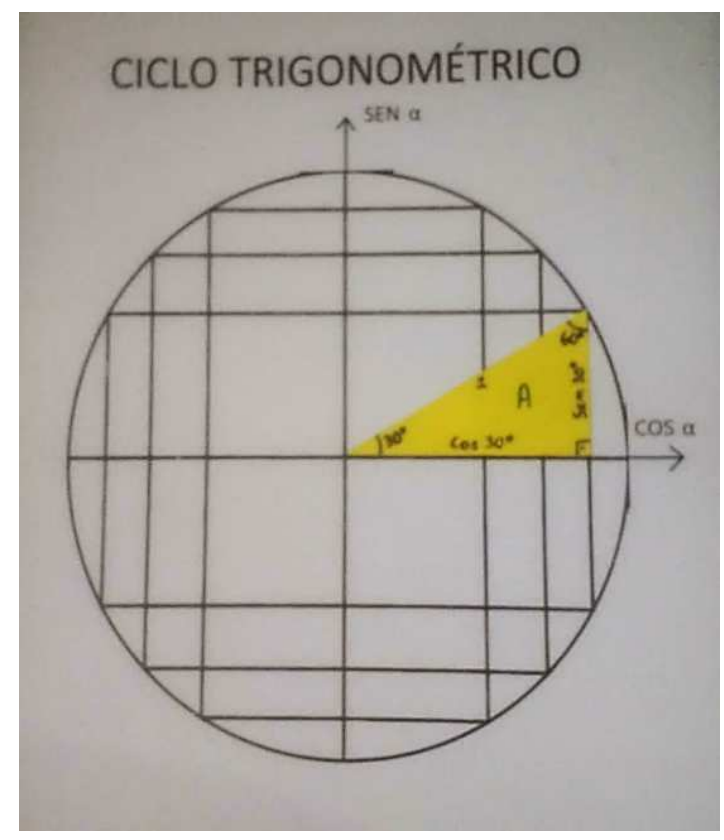

Fuente: Archivo personal de los investigadores (2020) 
En la Figura 4, está la hoja impresa con ciclo trigonométrico y el triángulo rectángulo A de catetos sen $30^{\circ} \mathrm{y} \cos 30^{\circ} \mathrm{e}$ hipotenusa 1 .

Figura 5. Ciclo trigonométrico con triángulo B

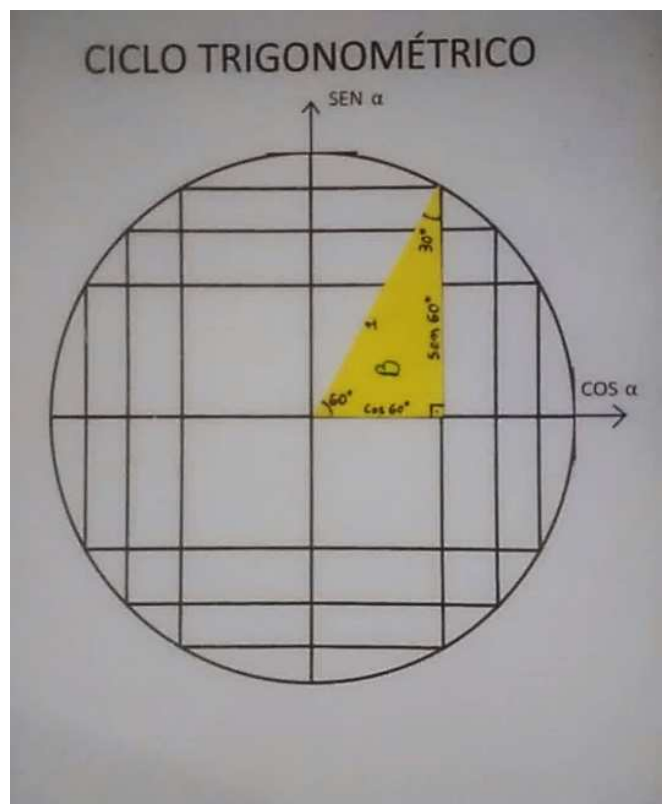

Fuente: Archivo personal de los investigadores (2020)

En la Figura 5, aparece el mismo ciclo trigonométrico, mostrado anteriormente, y el triángulo rectángulo $\mathrm{B}$ de catetos sen $60^{\circ} \mathrm{y} \cos 60^{\circ} \mathrm{e}$ hipotenusa 1 . Tenga en cuenta que el material es simple y se reproduce fácilmente sin utilización de muchos recursos financieros. Por ello, creemos que es ventajoso, por su relación costo-beneficio. Elegimos hacer los triángulos en color amarillo, usamos cartulina y los nombramos triángulo A y B para facilitar la distinción y ayudar en el uso de la guía. A pesar de que la reunión fuera extensa, no fue agotadora, ya que fue dividida entre todos las tareas de elaboración de los materiales. Creemos que esto ocurrió porque hubo, entre los participantes, un liderazgo compartido, apoyo y corresponsabilidad en las actividades desarrolladas.

Luego del desarrollo de la clase por parte del estudiante de licenciatura Gustavo, bajo la supervisión de la profesora Mariana, tuvimos dos encuentros para la evaluación de los participantes, siendo el cuarto encuentro, que se llevó a cabo el 26 de noviembre del año 2018, en el que estuvieron presentes el estudiante de licenciatura Gustavo y la investigadora, y el quinto encuentro, que se realizó el 11 de diciembre del año 2018 con la profesora Mariana y la investigadora, más detallados en el apartado de las evaluaciones de estos participantes. 


\section{Evaluaciones del estudiante de licenciatura Gustavo y la profesora Mariana sobre su participación en el trabajo de campo}

En este punto, primero mostraremos la información de la entrevista realizada al estudiante de licenciatura Gustavo y, posteriormente, de la entrevista a la Profesora Mariana.

Al preguntarle al estudiante de licenciatura cómo evalúa las experiencias de la investigación desarrolladas en el LEM FaE, expresó:

Estudiante de licenciatura Gustavo: Acerca del LEM, creo que fue fundamental para el diseño que hicimos. El material en sí nos trajo la idea para hacer el plan de clase. Nos basamos en lo que teníamos en el material, en la oportunidad que pudimos tener y, a través de ella, elaboramos el plan de clase y logramos alcanzar las metas propuestas. Entonces creo que LEM fue fundamental. El material en sí es muy bueno y estaba en excelentes condiciones. Entonces, estuvo bien.

(Archivo de audio del investigador, 2018)

El estudiante de licenciatura Gustavo evaluó positivamente las experiencias de la elaboración del plan de clases, destacando también que la alianza entre los involucrados (estudiante de licenciatura, profesora supervisora e investigadora) contribuyó a su elaboración. Afirmó que las experiencias del desarrollo del plan de clases fueron positivas para él y para los estudiantes, quienes tuvieron una buena implicación con las actividades.

Él también consideró que las experiencias de elaboración del plan de clase y del desarrollo de la clase en alianza con la profesora, fueron colaborativas según su análisis y resaltó que tanto la profesora supervisora como la investigadora le permitieron tener autonomía y lo apoyaron durante todo el proceso, lo cual indica el liderazgo compartido, apoyo y respeto mutuo (Fiorentini, 2004). Él no destacó ningún punto negativo. Cuando se le preguntó si volvería a participar y por qué, el estudiante de licenciatura Gustavo respondió:

Estudiante de licenciatura Gustavo: Sí participaría. Porque, fue un proceso de muy buena experiencia para mí. Tuve contacto tanto contigo como con Mariana. Pude aprender mucho sobre cómo ser profesor. Lo que yo tengo que hacer y lo que no tengo que hacer. Los materiales con los que pude contar son materiales que aportan mucho. Creo que es algo que incluso si pudiera dar una clase similar, volvería a darla nuevamente. Y también la oportunidad de estar en un espacio, de poder enseñar, en el que las personas estaban interesadas en lo que yo podría decir y por lo que yo podría pasar. Volvería a participar.

(Archivo de audio del investigador, 2018) 
Respecto a las experiencias desarrolladas en el trabajo de campo, el estudiante de licenciatura Gustavo manifestó que tuvieron un significado positivo tanto para su formación como para su práctica. Añadió que, como él aún no tenía experiencia como profesor, aprendió muchas cosas relacionadas con la actitud deseable del profesor y porque tuvo la oportunidad de enseñar de una forma diferente. También destacó la importancia de haber tenido acceso a nuevos materiales y aprendizajes relacionados con la didáctica. Lo cual se refiere a elementos de la cuarta concepción de Pimenta y Lima (2012), la pasantía como investigación, como alternativa para la formación de profesores, destacando lo que las autoras mencionan en relación a la "ampliación y análisis de los contextos donde se realizan las pasantías" (p. 46) y cuando menciona "la posibilidad de los pasantes de desarrollar posturas y habilidades de investigador"'(p. 46).

Frente al análisis del estudiante de licenciatura Gustavo, respecto a las experiencias en función de sus expectativas, manifestó que tenía un deseo inicial de conocer los materiales y saber si ellos podrían ayudar en el proceso de elaboración del plan de clase. Según él, las experiencias lograron suplir este deseo inicial. Al final de la entrevista, el estudiante de licenciatura Gustavo no hizo sugerencias para mejorar el trabajo colaborativo entre la investigadora y el estudiante dentro del alcance de la investigación. Simplemente elogió los momentos de apoyo y destacó el deseo de que más personas tuvieran la oportunidad que él tuvo o que fuese divulgado lo sucedido en los encuentros.

En la entrevista de la profesora Mariana, ella evaluó que fue muy productivo participar en el trabajo de campo, ya que, en vista de la temática de la clase, ciclo trigonométrico, el material manipulativo contribuyó para una mejor participación de los estudiantes. Respecto al LEM FaE, consideró que tanto para su pasante, como para la clase y para ella, como profesora supervisora, la experiencia fue muy importante, sobre todo porque no tuvo contacto con el laboratorio durante su pregrado. Respecto al LEM FaE, la profesora agregó que:

Profesora Mariana: El laboratorio es un lugar con muchos recursos y explorarlos, para la enseñanza básica secundaria, fue muy gratificante. Porque generalmente solemos trabajar más con el laboratorio en los primeros años de la escuela primaria. Entonces, tener esta experiencia en la enseñanza básica secundaria fue muy productivo.

(Archivo de audio del investigador, 2018) 
La profesora Mariana expresó que las experiencias fueron diferentes a sus experiencias anteriores envolviendo el recibimiento de pasantes en su sala de clases. Según ella, a pesar de que otros pasantes habían traído novedades a las clases, esta vez fue más satisfactorio, destacando que hubo una dedicación mayor a la planificación y al desarrollo de la clase, además de la participación extra de la investigadora, detalles contribuyeron a una mayor participación de los estudiantes. La profesora Mariana manifestó que volvería a participar en el trabajo de campo, dado que las experiencias, tanto de los involucrados como con el uso del LEM FaE, generaron aportes a su clase. Esta evaluación refleja elementos de la tercera y cuarta concepciones presentes en Pimenta y Lima (2012), es decir, las actividades de pasantía comprometidas con el contexto escolar, con foco en el entorno social en el que se dan y la pasantía como investigación y alternativa para la formación del profesor.

Cuando se le preguntó qué significado tenía para ella las experiencias desarrolladas en la investigación, en el LEM y en sus clases, la profesora afirmó que el uso del LEM FaE le mostró una nueva mirada sobre las posibilidades para la enseñanza con el uso de material manipulativo. Ante esto, ella enfatizó que tuvo significado tanto para su continua formación cuanto como investigadora en el área de Educación Matemática. Al solicitarle a la profesora Mariana que evaluará su experiencia en función de sus expectativas iniciales, dijo:

Profesora Mariana: hasta el pasante decía asi: "Ah Mariana, usted está con muchas expectativas. Calma. No sé si mi clase será todo eso, no, hasta porque me gustó mucho el material". Creo él exploró mucho más el material de lo que planeamos. Los estudiantes realmente discutieron. Tuvieron muchas dudas durante las actividades, pero eran dudas esperadas que realmente queríamos que se produjera con el material. Todo para que ellos se vayan cuestionando en los grupos y preguntando. Nosotros fuimos interviniendo, como: ¿Qué opinas de eso? ¿Qué pasa si rotamos el triángulo así? ¿Qué tienes? Se dieron cuenta de que era realmente genial. Incluso ellos perdieron el miedo al ciclo trigonométrico y la trigonometría. También informaron que la experiencia fue muy positiva. Tomando tanto el material que se produjo como el ciclo más amplio, fue sensacional para ellos. Y el cuidado que tuvieron tanto con el material y con el ciclo mayor. Por lo tanto, se dieron cuenta de la importancia de preservarla para que otros la usen. En conclusión, superó mis expectativas y fue más allá de lo que esperaba. Tanto el pasante y yo estábamos muy felices, supongo por lo que me dijo. Estaba muy contento con la acción.

(Archivo de audio del investigador, 2018) 
Casi el final de la entrevista, la profesora sugirió, con miras a proyectos futuros e investigaciones en curso, que la investigadora esté presente durante el desarrollo de la clase, aunque la profesora Mariana explicó que no era el objetivo de la investigación, ya que fue muy interesante lo que surgió en la clase. Ella consideró que podría haber superado aún más las expectativas de la investigadora la participación en las clases. También sugirió escribir, en el futuro, un artículo sobre el papel del material manipulado en las clases para que más profesores tengan acceso a estas experiencias.

La profesora Mariana afirmó que notó influencias del LEM FaE en la planificación y desarrollo de propuestas en la clase, agregando que el laboratorio fue importante para ella, para el estudiante de licenciatura Gustavo y los estudiantes. Finalmente, agregó:

Profesora Mariana: El laboratorio no solo es importante en este tema del desarrollo, también creo en la formación del pasante. Porque es una experiencia que no tenemos. Si, desde la experiencia de la pasantía, tiene esta experiencia en el laboratorio, puede contribuir, mucho a su práctica. Sobre todo porque él (refiriéndose al estudiante de licenciatura Gustavo) llegó a la escuela diciendo que quería ser profesor del curso intensivo para entrar en la universidad. Hasta entonces su cabeza estaba direccionada para ese curso. Tenía mucha experiencia en eso, pero no en la sala de clases. Entonces, para él fue muy enriquecedor. Aunque tiene experiencia frente al tablero, pero dirigiendo una sala con 38 estudiantes, muy agitado y ocupado, fue diferente. Entonces creo que esta experiencia de abrirse a la investigación en el laboratorio es muy importante. Creo que, aunque la investigación no ocurra en los próximos semestres, llevar esta idea, al supervisor para que explore el Laboratorio de Enseñanza de Matemáticas y así planificar sus clases. Incluso porque, también tengo esta sensación de explorar más el laboratorio para planificar mis clases. Nosotros vemos los materiales, vemos los planeamientos y nos adaptamos al contexto del aula.

(Archivo de audio del investigador, 2018)

Así, terminamos la descripción de las entrevistas realizadas al estudiante de licenciatura Gustavo y a la Profesora Mariana. Ahora, procederemos destacando los alcances y limitaciones de la participación en el trabajo de campo de estos participantes.

\section{Alcance y limitaciones del uso del LEM FaE: prácticas supervisadas y de colaboración}

En el ámbito del uso del LEM FaE, notamos, en la descripción de los encuentros y en la evaluación de los participantes, que el uso del laboratorio alcanzó la segunda y tercera 
concepción, los usos de un LEM mostrado en el trabajo de Lorenzato (2009). Se pudo notar que el uso del LEM FaE sirvió para apoyar a los involucrados en la planificación, organización, prueba y análisis de la preparación de la clase, posibilitando también la elaboración de los materiales necesarios para la clase. Además, en la evaluación del estudiante de licenciatura Gustavo y de la Profesora Mariana, se enfatizó el rol que jugó el LEM FaE en la elaboración del plan de clases.

En cuanto al alcance del uso del LEM FaE en el contexto de la Pasantía Supervisada, fue posible percibir elementos que remiten a las dos concepciones de Pimenta y Lima (2012): la pasantía como aproximación a la realidad y actividad teórica; pasantías como investigación. Así, se pudo notar que, a lo largo de la participación del estudiante de licenciatura Gustavo y de la Profesora Mariana, las actividades desarrolladas tuvieron una preocupación con el contexto escolar por lo que las prácticas involucraron a los estudiantes. A partir de las percepciones derivadas de las entrevistas semiestructuradas, se observa que además de la clase desarrollada, se brindó protagonismo de los estudiantes, también hubo otra relación con las matemáticas para el estudiante de licenciatura Gustavo. Además, se notó, debido a las situaciones propuestas por la profesora Mariana, que el estudiante de licenciatura Gustavo puede tener una postura y desarrollar las habilidades de un investigador, acercándose a lo propuesto por Pimenta y Lima (2012) en la cuarta concepción de la pasantía.

Finalmente, pensamos que hubo un alcance en cuanto a la colaboración entre los participantes de la investigación, ya que se identificaron elementos en la descripción de los encuentros y en el análisis de los participantes, propuesto por Fiorentini (2013), como: liderazgo compartido, apoyo, respeto mutuo y corresponsabilidad en las acciones desarrolladas.

Respecto a las limitaciones, hubo momentos en que el LEM FaE no estuvo disponible para albergar los encuentros, pero pensamos que esto no se interpuso en el avance del trabajo de campo.

\section{Algunas consideraciones}

Aportando elementos de la participación de uno de los estudiantes que participó en el trabajo de campo de la investigación, y de su supervisora, con un enfoque en los usos del LEM FaE en la formación de profesores en el ámbito de la Pasantía Supervisada y de la 
colaboración, consideramos que en cuanto al alcance del LEM FaE, se pudo notar, a lo largo del trabajo de campo, que el espacio puede brindar acceso a diversos recursos del laboratorio, tales como: materiales, juegos, computadora, tijeras, reglas, entre otros. También hubo momentos de conversaciones y debates entre los involucrados, para ayudar en el proceso de preparación del plan de clases tales como: planificación, organización, pruebas y análisis, siendo posible también confeccionar los materiales necesarios para las clases.

Pensamos que el intercambio de correos electrónicos y mensajes desde la aplicación WhatsApp contribuyó a otros usos del LEM FaE al facilitar la comunicación y registrar los avances entre los participantes en el trabajo de campo. En un escenario ideal, podría haber más equipos que permitan la comunicación en tiempo real (computadoras, cámara, micrófono). Otro hecho que es importante destacar es el uso de materiales sencillos, que se reproducen fácilmente, sin gastar una gran cantidad de recursos económicos. Por ello, creemos que el uso de estos materiales resulta ventajoso, dado su costo beneficio y la facilidad de adaptación por parte de otros profesores.

En el contexto del alcance del LEM FaE en la asignatura de pasantías supervisadas, se notó, por las situaciones propuestas por la profesora Mariana, que el estudiante de licenciatura Gustavo pudo tener otra postura y desarrollar las habilidades de un investigador, acercándose a lo propuesto por Pimenta y Lima (2012) en la tercera y cuarta concepción de la pasantía. Además, se observó, en la evaluación del estudiante, que las actividades de trabajo de campo contribuyeron a su formación docente.

Retomando el ámbito de la colaboración, también se identificaron elementos, en la descripción de los encuentros y en el análisis de los participantes, propuesto por Fiorentini (2013), como liderazgo compartido, apoyo y respeto mutuo, corresponsabilidad y espontaneidad en la realización de las acciones desarrolladas, voluntariedad; identidad entre participantes y reciprocidad de aprendizaje.

\section{Referencias}

Bogdan, R. y Biklen, S. (1994). A Investigação Qualitativa em Educação. Porto: Porto Editora. Fiorentini, D. (2013). Pesquisar práticas colaborativas ou pesquisar colaborativamente? En: Borba, Marcelo de Carvalho; Araújo, Jussara de Loiola (org) Pesquisa Qualitativa em Educação Matemática. pp 53-85. Belo Horizonte: Autêntica Editora

Justo, E. B. (2015). Construção de atividades para o trabalho no laboratório de matemática. 
Dissertação de Mestrado em Ensino de Ciências e Matemática. Pontifícia Universidade Católica de Minas Gerais, Belo Horizonte, MG, Brasil.

Lorenzato, S. (org). (2009). O laboratório de ensino de matemática na formação de professores / Sergio Lorenzato (org). 2 ed. Rev. Campinas: Autores Associados.

Pimenta, S. G. y Lima, M. S. L. (2012). Estágio e Docência. São Paulo: Cortez.

Pinto, N. K. D. (2020). O uso do Laboratório de Ensino de Matemática na formação de licenciandos em matemática. Dissertação de Mestrado Profissional Educação e Docência. Universidade Federal de Minas Gerais, Belo Horizonte, MG, Brasil.

Oliveira, R. R. M. (2017). Laboratório na escola: possibilidades para o ensino de matemática e formação docente. Dissertação de Mestrado Profissional Educação e Docência. Universidade Federal de Minas Gerais, Belo Horizonte, MG, Brasil.

Rodrigues, F. C. (2012). Laboratório de educação matemática: descobrindo as potencialidades do seu uso em um curso de formação de professores. Dissertação de Mestrado em Ensino de Ciências e Matemática. Pontifícia Universidade Católica de Minas Gerais, Belo Horizonte, MG, Brasil. 